\title{
Local photoreceptor degeneration causes local pathophysiological remodeling of retinal neurons
}

\author{
Bristol Denlinger, ${ }^{1}$ Zachary Helft, ${ }^{1,2}$ Michael Telias, ${ }^{1}$ Henri Lorach, ${ }^{3}$ Daniel Palanker, ${ }^{3,4}$ \\ and Richard H. Kramer ${ }^{1,2}$ \\ 'Department of Molecular and Cell Biology and ²Vision Science Graduate Group, University of California, Berkeley, Berkeley, \\ California, USA. ${ }^{3}$ Hansen Experimental Physics Laboratory and ${ }^{4}$ Department of Ophthalmology, Stanford University, \\ Stanford, California, USA.
}

\begin{abstract}
Vision loss in age-related macular degeneration (AMD) stems from disruption of photoreceptor cells in the macula, the central retinal area required for high-acuity vision. Mice and rats have no macula, but surgical insertion of a subretinal implant can induce localized photoreceptor degeneration due to chronic separation from retinal pigment epithelium, simulating a key aspect of AMD. We find that the implant-induced loss of photoreceptors in rat retina leads to local changes in the physiology of downstream retinal ganglion cells (RCCs), similar to changes in RGCs of rodent models of retinitis pigmentosa (RP), an inherited disease causing retina-wide photoreceptor degeneration. The local implant-induced changes in RGCs include enhanced intrinsic excitability leading to accelerated spontaneous firing, increased membrane permeability to fluorescent dyes, and enhanced photosensitization by azobenzene photoswitches. The local physiological changes are correlated with an increase in retinoic acid receptor-induced (RAR-induced) gene transcription, the key process underlying retinal remodeling in mouse models of RP. Hence the loss of photoreceptors, whether by local physical perturbation or by inherited mutation, leads to a stereotypical set of pathophysiological consequences in RGCs. These findings implicate RAR as a possible common therapeutic target for reversing the signal-corrupting effects of retinal remodeling in both RP and AMD.
\end{abstract}

Conflict of interest: RHK is a founder of Photoswitch Therapeutics Inc., a company developing commercial uses for photoswitches.

Copyright: (c) 2020, American Society for Clinical Investigation.

Submitted: July 29, 2019

Accepted: December 12, 2019

Published: January 30, 2020.

Reference information: /CI Insight. 2020;5(2):e132114.

https://doi.org/10.1172/jici.

insight.132114.

\section{Introduction}

Age-related macular degeneration (AMD) is the leading cause of vision loss in people above 60 years of age (1). In AMD, the deposition of extracellular material, called drusen, locally disrupts retinal pigment epithelium (RPE) cells and destroys rod and cone photoreceptor cells in the central part of the retina, known as the macula. This leads to the occurrence of blind spots that impair high-acuity vision, including most notably the ability to read and recognize faces. The macula accounts for less than $2 \%$ of the area of the retina, making AMD a highly localized disease that compels the development of therapies constrained to act locally, without having side effects on the healthy peripheral retina. Understanding the underlying causes and developing treatments for AMD have been hindered by the lack of animal models for the disease. Because mice and rats have a rod-dominant retina without a macula or a fovea, they cannot develop AMD. Larger mammals, including nonhuman primates, have cone-dominant retinas with a macula and fovea similar to humans, but spontaneous photoreceptor loss characteristic of AMD has not yet been found in species other than humans.

In contrast to AMD, there are many strains of mice, rats, and other mammals with mutations that cause photoreceptor degeneration syndromes very similar to human retinitis pigmentosa (RP). Rodent models of RP have revealed that degeneration of the photoreceptor layer is associated with changes in the morphology and physiology of the remaining layers of the retina. Late in the progression of RP, after the photoreceptors are lost, the retina exhibits morphological remodeling, including sprouting of new dendritic projections, formation of new ectopic synapses, migration of cells, and disruption of stratification (2-4). Physiological changes begin to occur earlier in the disease, while the photoreceptors are still deteriorating (5). Neurons in the outer (6) and inner retina (7-9) exhibit synchronous spontaneous oscillations 
of membrane potential, owing to increased gap-junctional coupling between neurons. Retinal ganglion cells (RGCs) become more permeable to large cations, owing to increased expression of P2X receptors, ionotropic receptors for extracellular ATP (10). RGCs also become intrinsically hyperactive because of increased expression of hyperpolarization-activated cyclic nucleotide-gated channels (10), a type of voltage-gated ion channel implicated in spontaneous pacemaker activity in the heart and brain. Together, these physiological changes lead RGCs to fire spontaneously in darkness at a much higher rate than in healthy retinas (5). Hyperactivity of RGCs can obscure light responses initiated by the remaining photoreceptors (11), a process that exacerbates vision impairment even before the photoreceptors are fully lost.

We recently discovered that retinoic acid (RA) is the trigger for pathophysiological changes in RGCs in rodent models of RP. The source of the elevated RA has not been firmly established, but many retinal cell types express retinaldehyde dehydrogenase (RALDH), an enzyme that synthesizes RA from the visual chromophore retinaldehyde (RAL). The loss of photoreceptor outer segments removes opsins that normally sequester a large amount of RAL. Hence the loss of photoreceptors may lead to more RAL substrate available for conversion to RA.

Our recent work (11) showed that the effects of RA on RGCs are mediated by the retinoic acid receptor (RAR), a DNA binding protein that regulates gene transcription. Pharmacological inhibition of RAR, as well as adenoassociated virus-mediated (AAV-mediated) gene therapy with a dominant-negative mutant of the $\alpha$ subunit of RAR, reduces hyperpermeability and hyperactivity. Inhibiting RAR in the retina of vision-impaired mice unmasks electrophysiological responses of RGCs to dim light and increases innate and learned light-elicited behaviors in vivo. These observations suggest that RAR-induced hyperactivity contributes to degrading visual performance as the photoreceptors are dying.

Do similar pathophysiological changes in RGCs occur in AMD, and if so, is RA again the trigger? Despite the lack of authentic animal models of AMD, local photoreceptor loss characteristic of AMD can be mimicked in rodents by photoablation (12) or by surgical implantation into the subretinal space of a piece of foreign material, such as a photovoltaic chip (13). The implant creates a chronic separation of photoreceptors from the RPE that results in local degeneration of photoreceptors but spares the inner layers of the retina, including the RGCs. Using the implant model, we tested whether local photoreceptor degeneration leads to heightened RA signaling and associated pathophysiological changes in RGCs.

\section{Results}

To induce degeneration of a local region of photoreceptors, we surgically inserted a nonresorbable implant in the subretinal space of the rat eye, detaching the photoreceptor outer segments from the RPE. The implant was $1 \mathrm{~mm}$ in diameter and $30 \mu \mathrm{m}$ in thickness, corresponding to approximately $1.6 \%$ of the retinal surface area (Figure 1A). For comparison, the diameter of the human macula and fovea are $5.5 \mathrm{~mm}$ and 1.5 $\mathrm{mm}$, corresponding to approximately $3 \%$ or $1 \%$, respectively, of the retinal surface area. Histological studies showed that subretinal implants locally eliminate all outer segments and nearly all rod and cone nuclei without disrupting inner retinal layers (13). We confirmed loss of photoreceptors with optical coherence tomography (OCT) imaging from live rats at 2 to 3 months after implantation (Figure 1, B and C).

In retinas from mouse and rat models of RP, elevated RA signaling can be detected in the ganglion cell layer (GCL) with an RAR-induced fluorescent protein reporter (11). The reporter construct has 2 tandem cassettes: red fluorescent protein (RFP), with expression controlled by a constitutive CMV promoter, and green fluorescent protein (GFP), with expression controlled by the RA response element (RARE), a DNA sequence that binds activated RAR. Binding of activated RAR to RARE enhances the action of the SV-40 promoter, which by itself is too weak to induce significant GFP expression. All cells infected by the virus express RFP, but only those cells exposed to RA express GFP. Hence the GFP/RFP ratio is proportional to RA-induced transcription controlling for viral transduction efficiency, which can vary between cells.

The reporter construct was delivered with an AAV expression vector, introduced by intravitreal injection. Within 2 to 3 weeks, transduced neurons exhibited red fluorescence, owing to expression of RFP (Figure 1D). Ordinarily, transduced neurons in healthy retinas express no detectable GFP (11). However, in GCL cells near the implant (within $200 \mu \mathrm{m}$ ), both RFP and GFP were often expressed (Figure 1, D and E). In contrast, in regions away from the implant (beyond $200 \mu \mathrm{m}$ ), GFP was absent. The GFP/RFP ratio was significantly higher over the degenerated area than in control areas from the same retinas $(0.43 \pm 0.03$ vs. $0.27 \pm 0.02, n=8$ retinal samples for each; $P<0.001)$. Hence local degeneration of photoreceptors leads to local elevation of RAR signaling in cells of the GCL layer. 
A

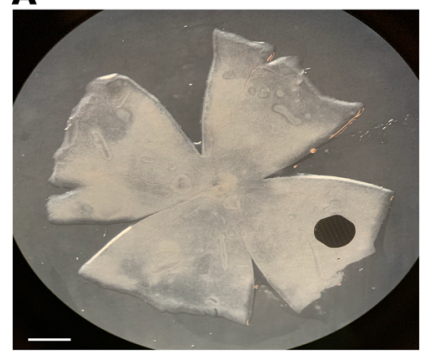

B

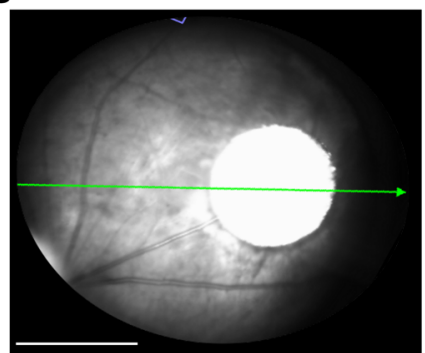

C

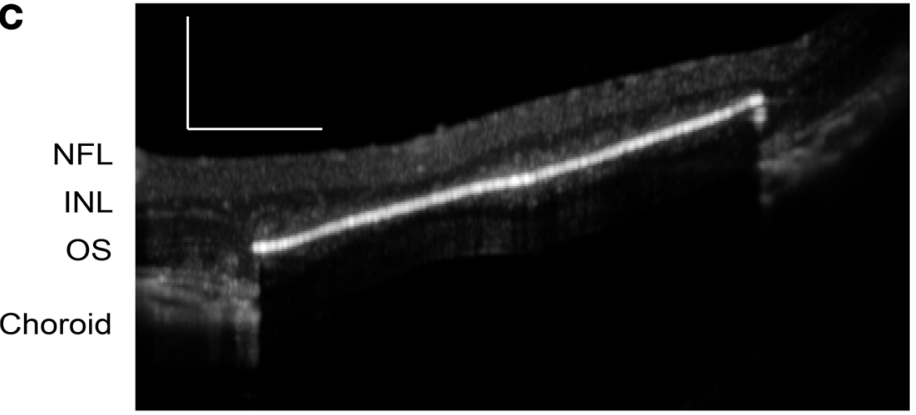

D RFP

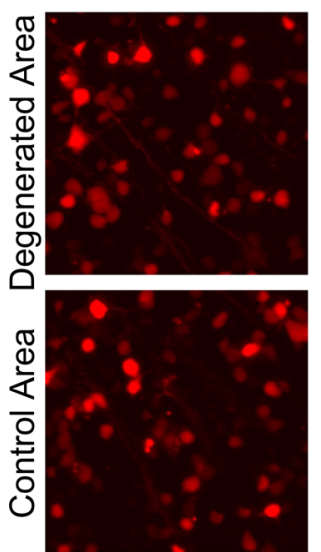

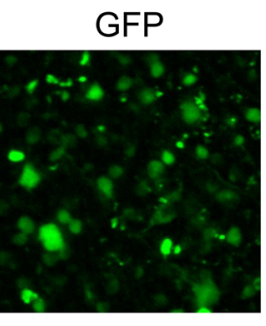

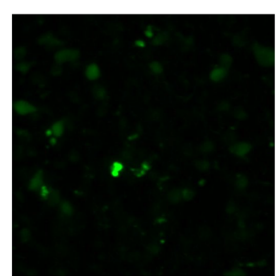

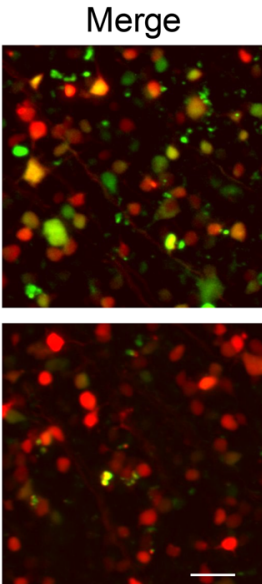

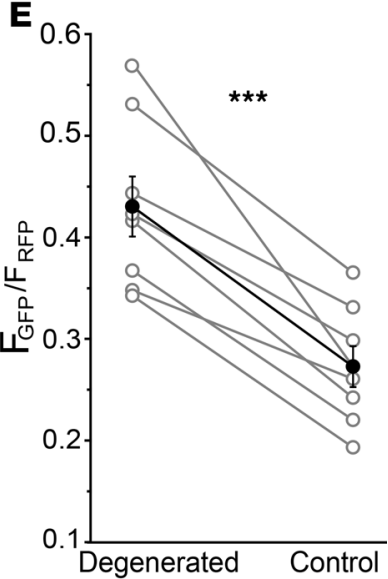

Figure 1. Implant-evoked photoreceptor degeneration induces local RA signaling. (A) Position of the subretinal chip in retina from an adult rat. Scale bar: $1 \mathrm{~mm}$. (B) Optical coherence tomography (OCT) fundus image of the chip implant in the intact rat eye. The green arrow represents the cross section in C. Scale bar: $1 \mathrm{~mm}$. (C) OCT image of the implant, demonstrating local loss of photoreceptor outer segments and cell bodies in the implanted region. Scale bar: $250 \mu \mathrm{m}$. NFL, nerve fiber layer; INL, inner nuclear layer; OS, outer segments. (D) Live fluorescence images of the retinoic acid receptor (RAR) reporter expressed in ganglion cell layer (GCL) cells. Red fluorescent protein (RFP) is expressed equally in the lesioned and nonlesioned area. Green fluorescent protein (GFP) is expressed more strongly near the lesion. Scale bar: $20 \mu \mathrm{m}$. (E) Quantification of GFP/RFP expression ratio. Data points represent mean GFP/RFP ratio with variability expressed as \pm SEM $(n=$ 8 retinal samples for each). ${ }^{* *} P<0.001$; paired $t$ test.

Elevated RA signaling induced by inherited photoreceptor degeneration brings about pathophysiological events, including membrane hyperpermeability and electrical hyperactivity in RGCs across the entire retina (10). We asked whether the local photoreceptor degeneration, induced by the subretinal implant, would cause similar pathophysiological events, but limited to RGCs near the implant. We started by analyzing spontaneous activity of RGCs in darkness (Figure 2). We used MEA recordings from retinas isolated from implanted rats, with the degenerated region straddling the edge of the array. RGCs near the implanted area (within $200 \mu \mathrm{m}$ of the edge) showed a nearly 10-fold higher spontaneous firing rate than RGCs far from the implant (beyond $200 \mu \mathrm{m}$ ), similar to the 8-fold higher spontaneous firing rate we observed when comparing photoreceptor-degenerated rd1 mouse retinas to WT mouse retinas (11). Addition of neurotransmitter receptor antagonists to block synaptic transmission mediated by glutamate, GABA, glycine, and acetylcholine eliminated firing of most RGCs far from the lesioned area but spared high-frequency firing in some 
A

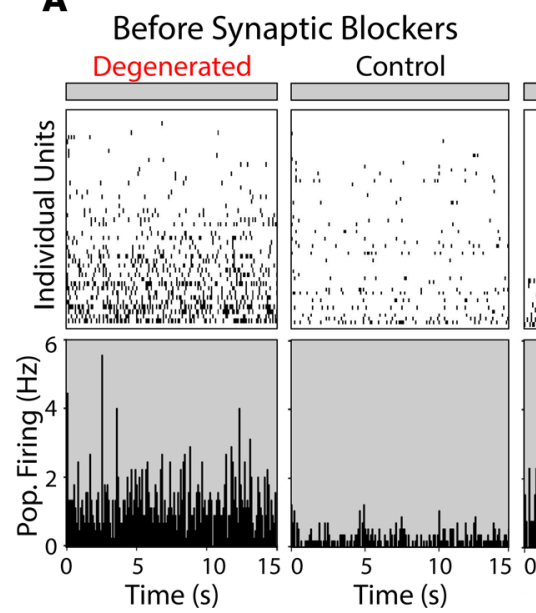

After Synaptic Blockers

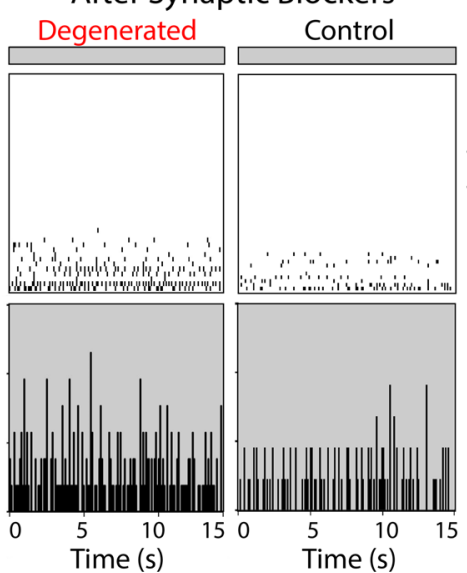

B

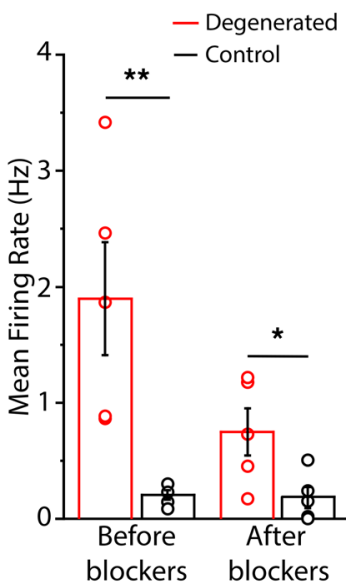

Figure 2. Implant-evoked photoreceptor degeneration induces local hyperactivity of RGCs. (A) Multi-electrode array (MEA) recordings before (left) and after (right) adding neurotransmitter receptor blockers, in degenerated (red) and control (black) samples taken from implanted retinas. (B) Quantification of spontaneous dark activity for degenerated ( $n$ $=5)$ and control $(n=5)$ retinal samples. Values represent mean firing rate $\pm S E M .{ }^{*} P<0.05 ;{ }^{* *} P<0.01$; 2-tailed $t$ test.

RGCs near or under the implant. This is consistent with the hyperactivity being intrinsic to RGCs (7, 8, 14, 15), with possible contributions from electrically coupled inner retinal neuron partners (16-18).

We next examined the effect of the azobenzene photoswitch BENAQ $(10,19)$ on implanted retinas. Photosensitization by BENAQ is degeneration dependent, a remarkable feature in which light sensitivity is conferred on RGCs in retinas with inherited global photoreceptor degeneration but not on RGCs from WT retinas with intact rods and cones (20). To test whether this feature also applies to local degeneration, we positioned the implanted region to straddle the edge of the MEA, and we compared the effect of BENAQ on RGCs near or far from the implant.

These experiments used a 3-step procedure. First, we measured the native light response to a saturating flash ( $1 \mathrm{~mW}, 15$ seconds). The flash elicited responses in only a few RGCs under the implant, versus many RGCs more than $200 \mu \mathrm{m}$ from the edge of the implant, consistent with local disruption of photoreceptors (Figure 3, A and B). The light response index (LRI) (21) was significantly smaller in RGCs near the implant (within $200 \mu \mathrm{m}$ ) than farther away (beyond $200 \mu \mathrm{m})(0.11 \pm 0.03, n=9$ retinal samples vs. $0.23 \pm 0.04, n$ $=15$ retinal samples; $P<0.05 ; 2$-tailed $t$ test). Second, we applied a mixture of neurotransmitter receptor antagonists to eliminate the contribution of rods and cones to the RGC light response. Under these conditions, light-evoked firing of RGCs was nearly completely eliminated. Intrinsically photosensitive RGCs were rare, consistent with infrequent melanopsin-expressing RGCs in rat retina (22). Third, we measured sensitivity to azobenzene photoswitches, by treating the retina with BENAQ for 30 minutes, followed by a rinse with BENAQ-free solution. BENAQ enters hyperpermeable RGCs through P2X receptors (10) and intracellularly blocks voltage-gated ion channels, so photosensitization of RGCs persists after rinsing, measurable as an increase in the action potential firing rate in response to illumination ( $1 \mathrm{~mW}, 15$ seconds). BENAQ treatment resulted in the emergence of light responses in RGCs near or under the implant (within $200 \mu \mathrm{m}$ ) but not in RGCs far from the implant (beyond $200 \mu \mathrm{m})($ LRI near implant $=0.24 \pm 0.02, n=12$ retinal samples vs. away from implant $=0.06 \pm 0.02, n=10$ retinal samples; $P<0.05$ ) (Figure $3, \mathrm{C}-\mathrm{F}$ ).

Inherited degeneration leads to RA-induced upregulation of $\mathrm{P} 2 \mathrm{X}$ receptors, which underlies hyperpermeability to large molecules, including the fluorescent dye YO-PRO-1, which binds DNA. We asked whether local photoreceptor degeneration, induced by the implant, would lead to local labeling of cells near the implant. Freshly isolated retinas were stained with YO-PRO-1 for 15 minutes before analysis. We compared the number of cells stained with YO-PRO-1 to the total number of cells, elucidated by labeling with NUCLEAR-ID.

Cells in the GCL showed bright YO-PRO-1 labeling near the implanted area (within $200 \mu \mathrm{m}$ ), especially around the perimeter. In contrast, cells far from the implant $(>200 \mu \mathrm{m})$ showed less labeling (Figure 4, A and B). The percentage of cells labeled with YO-PRO-1 was almost twice as great near the implant (within $200 \mu \mathrm{m}$ ) than far from the implant (beyond $200 \mu \mathrm{m})$ (Figure $4 \mathrm{C}),(10.6 \% \pm 1.9 \%$ vs. $20.9 \% \pm 4.4 \%, n=4$ retinal samples for each; $P<0.05$, paired $t$ test). By focusing through the retina we 
A
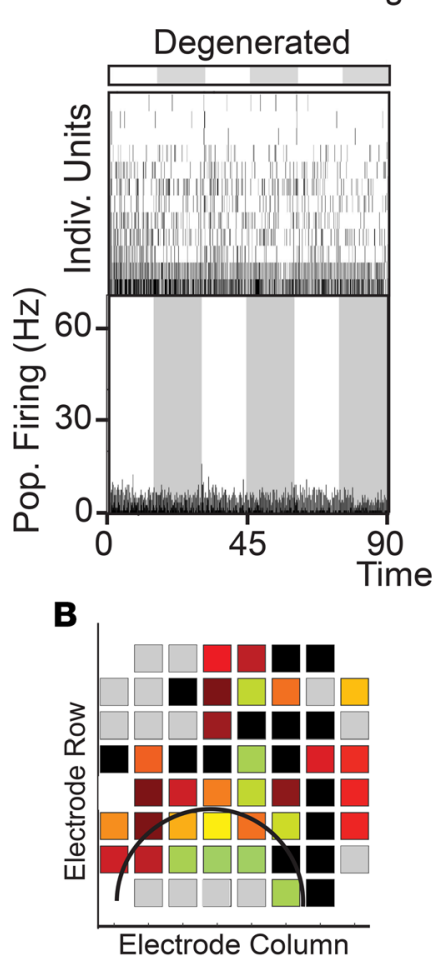

E

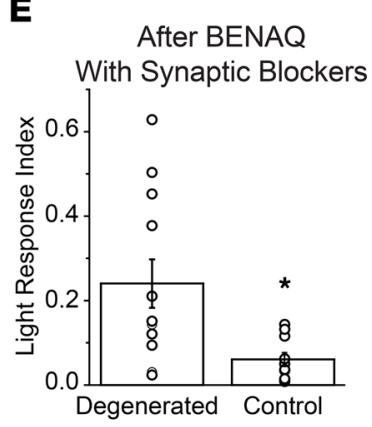

C BENAQ-mediated Light Response (after synaptic blockers)
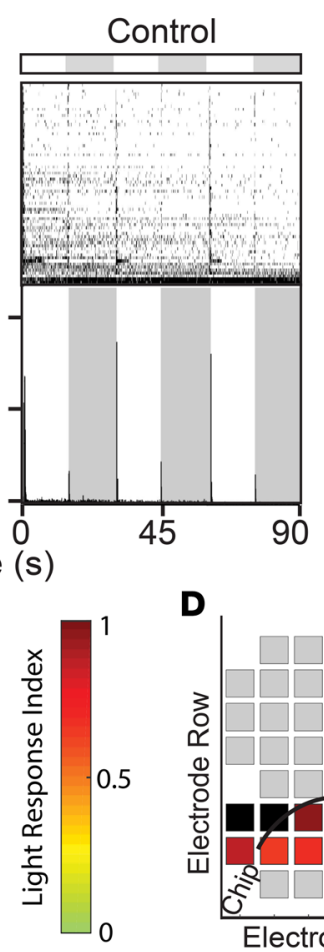

D
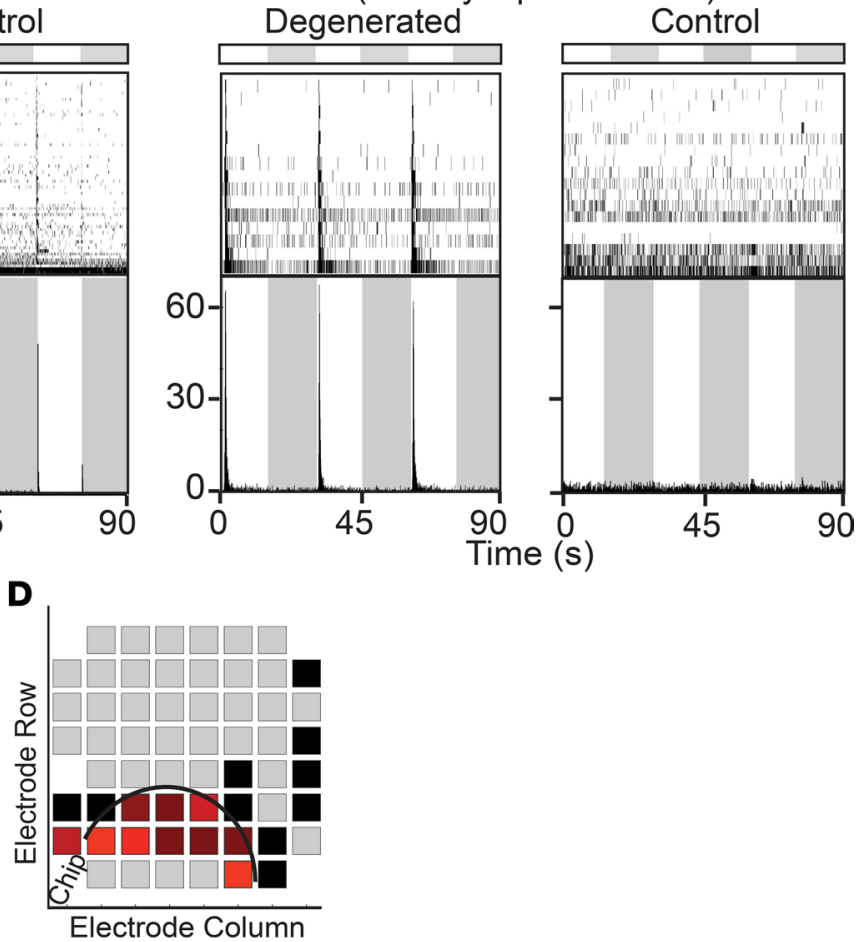

F

Degenerated Area

With Synaptic Blockers

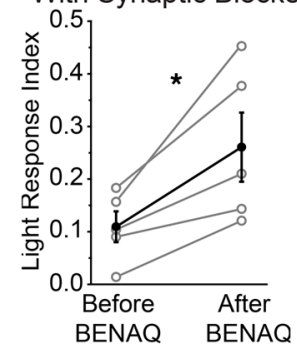

Figure 3. Implant-evoked photoreceptor degeneration induces localized BENAQ photosensitization of RGCs. (A and C) MEA recordings from control and degenerated samples, measuring both native photoreceptor-elicited light responses (A) and BENAQ-mediated light responses (C). Flash intensity was $1.0 \mathrm{~mW}$. (B and $\mathbf{D})$ Each square represents an individual MEA electrode and its native (B) or BENAQ-mediated (D) light response. Color scale shows light response index (LRI) for the units recorded by each electrode. Black squares represent electrodes with very low frequency spike activity $(<0.1 \mathrm{~Hz})$ but no response to light. Gray squares represent electrodes with no detectable activity. (E) LRI of degenerated $(n=12)$ or control $(n=10)$ regions. ${ }^{*} P<0.05$; 2-tailed $t$ test. (F) Responses from degenerated retina comparing the LRI before and after treatment with BENAQ and then after blockade of synapses with neurotransmitter antagonists $(n=5)$. Values are mean \pm SEM. ${ }^{*} P<0.05$; paired $t$ test.

could see that the area of YO-PRO-1 labeling aligned with the implant but extended for 200 to $250 \mu \mathrm{m}$ beyond the edge of the degenerated area (Figure 4, B and D). This is consistent with a diffusible signal emanating from the degenerated area that induces hyperpermeability in RGCs.

\section{Discussion}

Inherited retinal degeneration leads to RA-dependent gene transcription and global pathophysiological changes in RGCs (11). Our new findings indicate that the same process unfolds when degeneration is induced by local physical disruption of photoreceptors. The degeneration-induced phenotype can be mimicked in healthy mice by injecting either RA or its upstream precursor, RAL (11). This suggests that an overabundance of RAL might underlie the increase in RA signaling in degenerating retinas. The 
A Degenerated Area
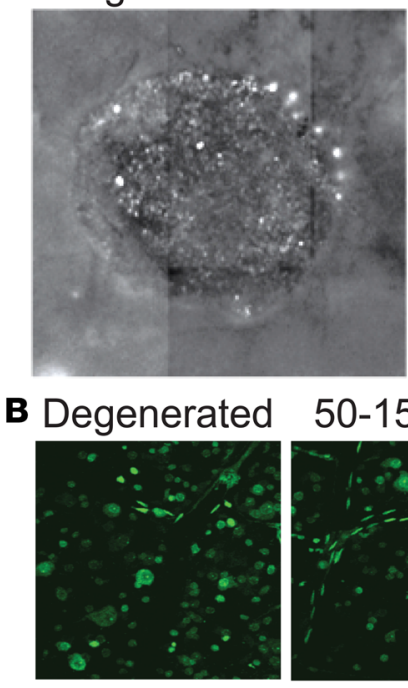

C

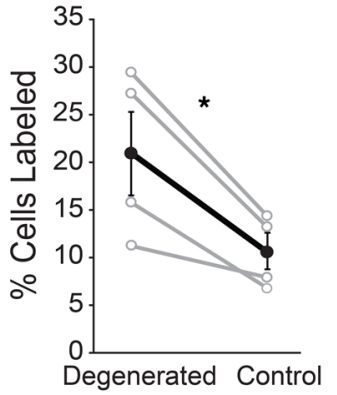

Control Area
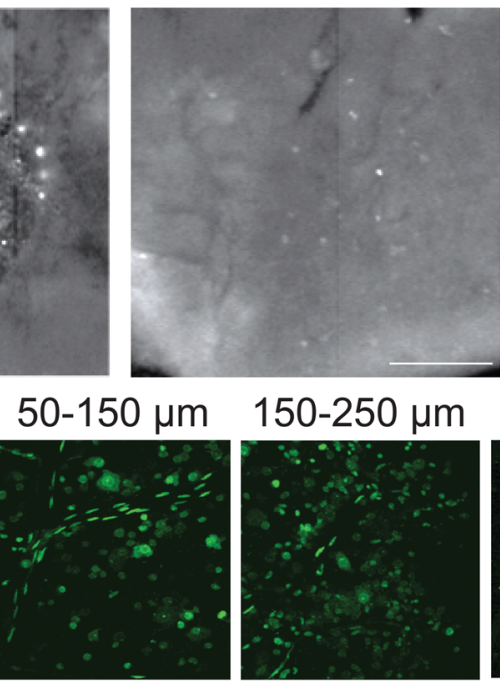

D 3.0

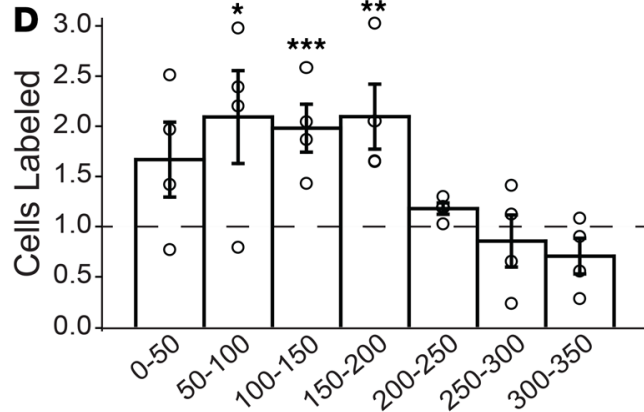

Distance From Implant ( $\mu \mathrm{m})$

Figure 4. Implant-evolked photoreceptor degeneration induces local hyperpermeability of GCL cells. (A) Composite wide-field fluorescence image showing multiple adjacent fields of view of an implanted flat-mounted retina stained with YO-PRO-1. Image shows punctate and diffuse labeling in degenerated area (left) and less punctate labeling in control area (right). Scale bar: $0.5 \mathrm{~mm}$. (B) Higher magnification fluorescence images of YO-PRO-1 labeling of individual GCL cells. Images are $2 \mathrm{D}$ projections of $Z$ stacks. Note that few cells are labeled beyond $300 \mu \mathrm{m}$ from the implant. Scale bar: 50 $\mu \mathrm{m}$. (C) Group data of YO-PRO-1 labeling near the implant (within $200 \mu \mathrm{m}$ of the implant) versus a control area, less than $1000 \mu \mathrm{m}$ from implant ( $n=4$ areas for each). ${ }^{*} P<0.05$; paired $t$ test. (D) Decline of YO-PRO-1 labeling with distance from the edge of the implant. $n=4$ retinal samples. ${ }^{*} P<0.05$; ${ }^{*} P<0.01$; ${ }^{* *} P<0.001$; 1-way ANOVA with Tukey's honestly statistical difference (HSD) post hoc test. Values are mean \pm SEM.

enzymatic machinery necessary for synthesizing RAL is expressed only in RPE, rods and cones, and Müller glial cells (23), but RALDH is expressed in nearly all retinal neurons $(24,25)$. Hence, in principle, enhanced RA signaling in RGCs could result from the diffusion of either the substrate RAL, or the product RA, from the outer to the inner retina. RAL is normally sequestered in photoreceptor outer segments by opsins, which are present in great abundance (100 million copies of rhodopsin per rod). The degeneration of photoreceptors eliminates this enormous molecular sink, allowing more RAL and/or more RA to diffuse through the neural retina to RGCs. In addition, the loss of photoreceptors could breach the outer limiting membrane, facilitating spread through the retina. Our fluorescent reporter indicates that RAR-induced transcription is activated in cells near the implant. Downstream pathophysiological events, including hyperactivity and hyperpermeability, also occur near the implant. YO-PRO-1 labeling indicates that hyperpermeability extends up to $200 \mu \mathrm{m}$ beyond the edge of the implant, consistent with vectorial diffusion of RAL and/or RA.

Our measurements have focused on RGCs. However, other retinal neurons also show pathophysiological changes in rodent models of RP, including heightened spontaneous synaptic release from bipolar cells (26) and heightened electrical coupling between inner retinal neurons, promoting spontaneous oscillations $(16,18)$. These changes might also occur with local photoreceptor degeneration and may also involve locally elevated RA signaling. 
Both the hyperpermeability and hyperactivity of RGCs could have deleterious consequences on remaining visual function as the photoreceptors die. The P2X receptors that mediate RGC hyperpermeability conduct $\mathrm{Ca}^{2+}$. Chronic activation of $\mathrm{P} 2 \mathrm{X}$ receptors can result in a sustained increase in intracellular $\mathrm{Ca}^{2+}$, which can trigger physiological and morphological neuronal plasticity (27), and aberrant rewiring of the retinal circuit. Hyperactivity can corrupt information processing in RGCs by obscuring light-elicited responses that are initiated by the remaining photoreceptors (17). Hyperactivity might occur whenever a local region of photoreceptors is affected, for example in AMD, but this remains to be determined. In principle, a spatially restricted lesion of photoreceptors can have a halo effect, generating a broader region of RGCs that exhibit hyperpermeability and hyperactivity, exacerbating and enlarging the region of visual deficit. In this scenario, treatments that reverse RGC pathophysiology might actually shrink a visual scotoma, even if the central region of the scotoma is completely devoid of photoreceptors, such as in the geographic atrophy occurring in advanced nonexudative AMD.

We have shown that inhibitors of RAR can reverse RGC hyperpermeability and hyperactivity and improve light sensitivity in slowly degenerating retinas from the rd10 mouse strain (11). RAR can be inhibited pharmacologically, with a high-affinity drug candidate such as BMS-493, or genetically, with an AAV vector that encodes a dominant-negative mutant of the $\alpha$ subunit of RAR. Because RAR is activated only near sites of photoreceptor degeneration, drug or gene therapy should alter function near the degenerated region while having few or no side effects on healthy areas of the retina. By unmasking small signals buried in the noise of RGC hyperactivity, inhibition of RAR may not only aid in resolving naturally transduced light signals from damaged retinas but also augment artificially transduced light signals. This includes those generated by optoelectronic or optogenetic tools, by photoreceptors regenerated from stem cells, or by photoswitches such as BENAQ. RAR inhibitors might be particularly effective in improving prosthetic vision enabled by a subretinal optoelectronic implant, which stimulates inner retinal neurons in the scotoma of patients with advanced AMD (28).

\section{Methods}

Subretinal implantation. WT Long-Evans rats from Charles River (Wilmington, Massachusetts, USA) were housed with a 12-hour light/12-hour dark cycle. Implants were introduced as described previously (29). WT rats (P35-P50) were anesthetized with ketamine $(75 \mathrm{mg} / \mathrm{kg})$ and xylazine $(5 \mathrm{mg} / \mathrm{kg})$, delivered by intramuscular injection. The sclera and choroid were incised, and saline was injected to create a retinal detachment. Implants (30 $\mu \mathrm{m}$ thick, $1 \mathrm{~mm}$ in diameter) composed of SU8 parylene or aluminum foil were inserted into the subretinal space, positioned 2 to $4 \mathrm{~mm}$ from the optic disc. The incision was then sutured and treated with a local antibiotic (bacitracin/polymyxin B). Postsurgical outcome was assessed in vivo with OCT (SPECTRALIS, Heidelberg Engineering).

Tissue preparation. Rats were euthanized with an overdose of isoflurane followed by cardiac puncture. Eyes were obtained from rats immediately following euthanasia. Retinas were removed and kept in saline containing (in mM) $119 \mathrm{NaCl}, 2.5 \mathrm{KCl}, 1 \mathrm{KH}_{2} \mathrm{PO}_{4}, 1.3 \mathrm{MgCl}_{2}, 2.5 \mathrm{CaCl}_{2}, 26.2 \mathrm{NaHCO}_{3}$, and 20 D-glucose, aerated with $95 \%: 5 \% \mathrm{O}_{2} / \mathrm{CO}_{2}$ at room temperature. Dissection was performed under ambient light conditions. Typically, the implant area was divided into 2 retinal pieces ( $n=2$ for each retina).

$M E A$ recordings. Retinas were mounted on a 60-electrode Multi-Electrode Array (MEA 1060-2-BC, Multi-Channel Systems) and perfused with oxygenated saline at $34^{\circ} \mathrm{C}$. Recordings were high-pass filtered at $200 \mathrm{~Hz}$ and digitized at $20 \mathrm{kHz}$. We used a criterion of $4 \mathrm{SD}$ above the mean background voltage signal for spike detection. Typically, each electrode recorded spikes from 1 to 3 RGCs. Principal component analysis was used for sorting spikes from different cells (Offline Sorter, Plexon). Stimulation light was generated from a 100-W mercury arc lamp (Ushio USH-103D), and neutral density filters were used to reduce intensity. LRI was defined as the absolute value of the mean firing rates in light - dark divided by mean firing rates in light + dark $(20,21)$. The solution for synaptic blockade contained (in mM) 10 AP4, 40 DNQX, 30 AP5, 10 SR-95531 (gabazine), 50 TPMPA, 10 strychnine, and 50 tubocurarine.

Imaging. Retinas were flat-mounted on filter paper with the GCL side up and imaged with a spinning disk confocal microscope (Olympus BX61WI) equipped with a mercury lamp, a 40× water-immersion objective, and standard GFP (488/519 nm) and RFP (561/575 nm) filter cubes (Olympus, U-URA). Three-dimensional $Z$ stacks (1.5 $\mu \mathrm{m}$ /section) were acquired using a Hamamatsu ImagEM-CCD C9100-13. Images were analyzed with ImageJ (NIH). For dye-loading experiments, flat-mounted retinas were stained with $500 \mathrm{nM}$ YO-PRO-1 (Life Technologies) in oxygenated saline for 15 minutes. To reveal all nuclei in the field of view, we counterstained with NUCLEAR-ID (Enzo Life Sciences) at a 1:500 dilution for 3 minutes. 
Unincorporated dye was removed by washing with normal saline. Overlapping nuclei and those belonging to vascular pericytes (aspect ratio $>1.5$ ) were excluded.

For RAR-dependent gene expression experiments, we used a genetically encoded dual RFP/GFP reporter described previously (11). An AAV2 vector encoding the reporter was injected intravitreally (8-10 $\mu \mathrm{L}$ in each eye) at 2 to 3 months after subretinal implantation, and retinas were imaged at 2 to 3 weeks after injection. For analysis, we selected single-cell regions of interest from $2 \mathrm{D}$ projections of $Z$ stacks, first on the RFP channel and then on the GFP channel.

Statistics. Measurements are represented as the mean \pm SEM. For comparisons between 2 data sets, a normality test was used, followed by a 2-tailed $t$ test or a paired $t$ test when appropriate. For comparisons between multiple groups, a 1-way ANOVA followed by a Tukey's HSD post hoc test was used. Statistical significance was set at $P<0.05$. ${ }^{*} P<0.05 ; * * P<0.01 ; * * * P<0.001$.

Study approval. All animal use procedures were approved by the University of California, Berkeley, Animal Care and Use Committee or in accordance with the institutional guidelines at Stanford University and conformed to the Statement for the Use of Animals in Ophthalmic and Vision Research of the Association for Research in Vision and Ophthalmology.

\section{Author contributions}

$\mathrm{BD}$ designed and performed all MEA experiments, wrote the manuscript, and prepared the figures. $\mathrm{ZH}$ carried out YO-PRO-1 imaging and wrote the manuscript. MT developed the virus, conducted imaging experiments, and wrote the manuscript. HL performed subretinal chip implantation. DP designed experiments and supervised chip implantation. RHK designed experiments, wrote the manuscript, and supervised the project. All authors reviewed the manuscript.

\section{Acknowledgments}

This work was supported by grants from the National Eye Institute (R01EY024334, R24EY023937, and P30EY003176), the Thome Foundation, and the Foundation for Fighting Blindness. DP and HL were supported by the NIH (R01-EY-018608, R01-EY-027786), the Department of Defense (W81XWH-15-1-0009), the Stanford Institute of Neuroscience, and Research to Prevent Blindness.

Address correspondence to: Richard H. Kramer, 121 Life Science Building, Department of Molecular and Cell Biology, University of California, Berkeley, Berkeley, California 94720-3200, USA. Phone: 510.407.7424; Email: rhkramer@berkeley.edu.

1. Wong WL, et al. Global prevalence of age-related macular degeneration and disease burden projection for 2020 and 2040 : a systematic review and meta-analysis. Lancet Glob Health. 2014;2(2):e106-e116.

2. Marc RE, Jones BW. Retinal remodeling in inherited photoreceptor degenerations. Mol Neurobiol. 2003;28(2):139-147.

3. Jones BW, et al. Retinal remodeling triggered by photoreceptor degenerations. J Comp Neurol. 2003;464(1):1-16.

4. Jones BW, Pfeiffer RL, Ferrell WD, Watt CB, Marmor M, Marc RE. Retinal remodeling in human retinitis pigmentosa. Exp Eye Res. 2016;150:149-165.

5. Stasheff SF, Shankar M, Andrews MP. Developmental time course distinguishes changes in spontaneous and light-evoked retinal ganglion cell activity in rd1 and rd10 mice. J Neurophysiol. 2011;105(6):3002-3009.

6. Haq W, Arango-Gonzalez B, Zrenner E, Euler T, Schubert T. Synaptic remodeling generates synchronous oscillations in the degenerated outer mouse retina. Front Neural Circuits. 2014;8:108.

7. Borowska J, Trenholm S, Awatramani GB. An intrinsic neural oscillator in the degenerating mouse retina. J Neurosci. 2011;31(13):5000-5012.

8. Trenholm S, et al. Intrinsic oscillatory activity arising within the electrically coupled AII amacrine-ON cone bipolar cell network is driven by voltage-gated $\mathrm{Na}+$ channels. J Physiol (Lond). 2012;590(10):2501-2517.

9. Margolis DJ, Gartland AJ, Singer JH, Detwiler PB. Network oscillations drive correlated spiking of ON and OFF ganglion cells in the rd1 mouse model of retinal degeneration. PLOS ONE. 2014;9(1):e86253.

10. Tochitsky I, et al. How azobenzene photoswitches restore visual responses to the blind retina. Neuron. 2016;92(1):100-113.

11. Telias M, Denlinger B, Helft Z, Thornton C, Beckwith-Cohen B, Kramer RH. Retinoic acid induces hyperactivity, and blocking its receptor unmasks light responses and augments vision in retinal degeneration. Neuron. 2019;102(3):574-586.e5.

12. Busch EM, Gorgels TG, van Norren D. Temporal sequence of changes in rat retina after UV-A and blue light exposure. Vision Res. 1999;39(7):1233-1247.

13. Lorach H, et al. Development of animal models of local retinal degeneration. Invest Ophthalmol Vis Sci. 2015;56(8):4644-4652.

14. Sekirnjak C, et al. Changes in physiological properties of rat ganglion cells during retinal degeneration. J Neurophysiol. 2011;105(5):2560-2571.

15. Yee CW, Toychiev AH, Sagdullaev BT. Network deficiency exacerbates impairment in a mouse model of retinal degeneration. 
Front Syst Neurosci. 2012;6:8.

16. Choi $\mathrm{H}$, et al. Intrinsic bursting of AII amacrine cells underlies oscillations in the rd1 mouse retina. J Neurophysiol. 2014;112(6):1491-1504

17. Ivanova E, Yee CW, Baldoni R, Sagdullaev BT. Aberrant activity in retinal degeneration impairs central visual processing and relies on Cx36-containing gap junctions. Exp Eye Res. 2016;150:81-89.

18. Toychiev AH, Ivanova E, Yee CW, Sagdullaev BT. Block of gap junctions eliminates aberrant activity and restores light responses during retinal degeneration. J Neurosci. 2013;33(35):13972-13977.

19. Mourot A, et al. Tuning photochromic ion channel blockers. ACS Chem Neurosci. 2011;2(9):536-543.

20. Tochitsky I, et al. Restoring visual function to blind mice with a photoswitch that exploits electrophysiological remodeling of retinal ganglion cells. Neuron. 2014;81(4):800-813.

21. Polosukhina A, et al. Photochemical restoration of visual responses in blind mice. Neuron. 2012;75(2):271-282.

22. Reifler AN, et al. The rat retina has five types of ganglion-cell photoreceptors. Exp Eye Res. 2015;130:17-28

23. Parker RO, Crouch RK. Retinol dehydrogenases (RDHs) in the visual cycle. Exp Eye Res. 2010;91(6):788-792.

24. Fischer AJ, Wallman J, Mertz JR, Stell WK. Localization of retinoid binding proteins, retinoid receptors, and retinaldehyde dehydrogenase in the chick eye. J Neurocytol. 1999;28(7):597-609.

25. Harper AR, Wiechmann AF, Moiseyev G, Ma JX, Summers JA. Identification of active retinaldehyde dehydrogenase isoforms in the postnatal human eye. PLOS ONE. 2015;10(3):e0122008.

26. Margolis DJ, Newkirk G, Euler T, Detwiler PB. Functional stability of retinal ganglion cells after degeneration-induced changes in synaptic input. J Neurosci. 2008;28(25):6526-6536.

27. Virginio C, MacKenzie A, Rassendren FA, North RA, Surprenant A. Pore dilation of neuronal P2X receptor channels. Nat Neurosci. 1999;2(4):315-321.

28. Goetz GA, Palanker DV. Electronic approaches to restoration of sight. Rep Prog Phys. 2016;79(9):096701.

29. Mandel Y, et al. Cortical responses elicited by photovoltaic subretinal prostheses exhibit similarities to visually evoked potentials. Nat Commun. 2013;4:1980. 